MATEC Web of Conferences 40, 01007 (2016)

DOI: $10.1051 /$ matecconf/20164001007

C) Owned by the authors, published by EDP Sciences, 2016

\title{
Spectral characteristics of Acoustic Emission of rock based on Singular point of HHT Analysis
}

\author{
ZHOU Xiaoshan ${ }^{1}$, LU Xin ${ }^{2}$,LIU Xiangxin ${ }^{3}$ \\ ${ }^{1}$ Continue Education College, North China of Science and Technology, Tangshan, Heibei 063009; \\ ${ }^{2}$ Modern technology and education centre, North China of Science and Technology, Tangshan, Heibei 063009; \\ ${ }^{3}$ College of Mining Engineering, North China of Science and Technology, Tangshan, Hebei 063009, China
}

\begin{abstract}
The sandstone test of uniaxial compression acoustic emission (AE) test has been studied, the HHT analysis is applied to AE signal processing, and through the analysis of AE signal to reveal the process of rock fracture. The results show that HHT is a method that based on principal component analysis of time-frequency analysis. The method of HHT can very convenient to deal the singular signal; it can be determine the main composition of singular signal. The instantaneous frequency can be used to describe precisely the time-frequency characteristics of singular signal. The method has a very important significance to reveal the frequency characteristics of AE signal. The EMD signal is decomposed into 8 IMF components in the failure process of rock sound. The component of IMF1 IMF4 is the main component, and the IMF5 IMF8 for low frequency noise signal. Through the EMD of AE signal frequency, the rock fracture has been decomposition into three stages: the initial zone, wave zone, quiet zone. This shows that in the analysis of rupture must eliminate noise interference signal characteristics of $\mathrm{AE}$.
\end{abstract}

\section{Introduction}

As we known, there is a process from quantitative change to qualitative change in the occurrence of any damage. Various size of energy will be produced by rock damage; also the phenomenon of $\mathrm{AE}$ will generated. Based on the research of the $\mathrm{AE}$ signal, the internal condition change and the inversion of the rock failure mechanism can be concluded $^{[1]}$

In recent years, with the development of $\mathrm{AE}$ technology, and its application in rock mechanics, many scholars use the AE testing technology for rock failure process carried on the thorough analysis and research, including: Mansurov and Rudajev predict rock damage types by the AE measurement ${ }^{[2-3]}$. Nomikos studied on uniaxial compression under Mourinho Dionysus marble, and found that the AE activity and the energy release were increased significantly when the crack damage maximum phase ${ }^{[4]}$. Ganne studied the peak before the brittle failure of rock by using the $\mathrm{AE}$ technology, 4 process of AE accumulated energy could be taken ${ }^{[5]}$. Wang have took an AE positioning research on the rotor friction united the wavelet transform and correlation analysis, and a conclusion that a higher position precision than directly related to positioning ${ }^{[6]}$. Ishida through the AE signal inversion location of rock failure ${ }^{[7]}$. Moriya have taken an study on broken evolution process in Bain salt mine in Germany, and found that the distribution of AE signal changes over principal stress rotation direction and depth ${ }^{[8]}$.
The main research is from parameter analysis, just only from qualitative to description the rock failure process. This is very limited to reflect the rock failure nature from the angle of $\mathrm{AE}$.

\section{Process}

\subsection{The test system}

The experimental system is composed of loading system and AE system. The loading system used the TAW-3000 microcomputer controlled electro-hydraulic servo process, the AE system used the PCI-2 AE system produced on America acoustic physical company. In order to avoid the noise of contacting, using the axial loading control and pre-loading to $1.5 \mathrm{kN}$, and followed by $0.2 \mathrm{~mm} / \mathrm{min}$ to failure. The threshold value of $\mathrm{AE}$ instrument set $40 \mathrm{~dB}$, the waveform settings in the sampling rate is $1 \mathrm{MSPS}$, the pre-trigger is 256 and the length is $2 \mathrm{~K}$. The sensor is the R6 $\alpha$ resonant type high sensitivity sensor, whose operating frequency is $35 \sim 100 \mathrm{kHz}$.Using the rock cutting mechanism to get the size of $50 \mathrm{~mm} \times 50 \mathrm{~mm} \times$ $100 \mathrm{~mm}$ (length $\times$ width $\times$ height), and the $\mathrm{AE}$ sensor probe directly fixed on the specimen surface.

\subsection{AE signal processing}

\subsubsection{The singular signal of $A E$}


The stress-time curve of sandstone under uniaxial compression experiment was shown in figure 1 . In order to use the AE to characterize the whole rupture process of rock, according to the mechanical curve of rock, select several signal point, represented by A, B, C, D and 1-7. According to point of $\mathrm{A}, \mathrm{B}$ and $\mathrm{D}$, the curve can divide into the following stages [12]: (1) the nonlinear compressive stage of $\mathrm{OA}$, take point 1 to make an analysis; (2) the stage of linear elastic deformation, represented as $\mathrm{AB}$, take point 2 and 3 to make an analysis; (3) the stage of nonlinear deformation stage, represented as $\mathrm{BC}$, it can be further divided into following stage: (1) The stage of stable crack growth $\left(\mathrm{BB}_{0}\right)$, take the point 5 to analysis. (2)Non-elastic deformation stage $\left(\mathrm{B}_{0} \mathrm{C}\right)$, take the point 6 and 7 to analysis, $\mathrm{C}$ point is called the peak stress $\left(\sigma_{\mathrm{c}}=34.36 \mathrm{MPa}\right)$; (4) the strain softening stage of $\mathrm{CD}$ segment, $\mathrm{C}_{0}$ is the sample completely failure point.

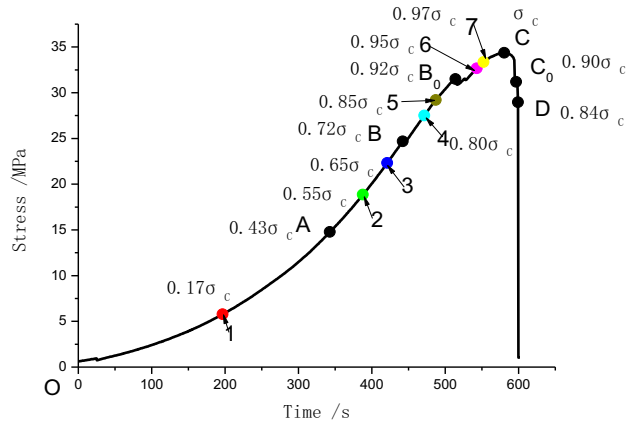

Figure 1.Rock stress-time curve and singular point selection

\subsubsection{AE signal processing}

Step 1: take the curve of AE signal under the MATLAB program. Step 2: the original signal of AE is decomposed into a series of different characteristic time scales of intrinsic mode function (IMF) by EMD decomposition of HHT and formula (1) and (2). Step 3: to calculate the contribution rate and the coefficient of original signal of each IMF component, and determine the advantages of the weight of IMF by principal component analysis and correlation analysis. Step 4: the advantage of the IMF component type (4) is used to calculate the Hilbert spectrum, type (5) is used to calculate the marginal spectrum, the graphics of Hilbert spectrum and marginal spectrum can be draw by these analyses.

\subsection{AE signal singularity analysis}

\subsubsection{Principal component analysis}

In principal component analysis, the variance contribution rate was used to measure the information content of someone factor. The IMF component was received by EMD decomposition, different components represent different factor, and the original signal component of main ingredients of the IMF can be determine by calculation of variance contribution ratio of the component.

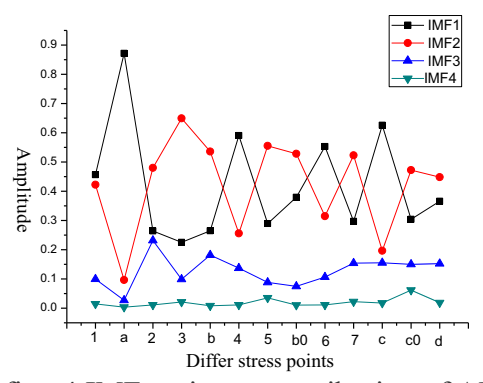

Figure 2. The first 4 IMF variance contribution of AE signal

From the Figure 2 can be seen that at the same stress level, the IMF1 IMF4 variance rate is relatively large by EMD decomposition, and it can reach more than $98 \%$. The IMF1 and IMF2 variance contribution rate changed with the stress obvious, the contribution rate of IMF3 and IMF4 components is very small.

\subsubsection{Correlation analysis}

The EMD decomposition with fixed basis function, and each IMF component extraction is determined by the nature of the signal, each IMF component is similar to the original signal. The correlation coefficient is used to reflect the degree of similar two signals in the correlation coefficient. According to the characteristics of the signal correlation, the number of relations between the noise component and the original signal should be zero. If a component with the original signal on the number of relations between each other is very small, it is likely to be noise component. Therefore, it can determine whether the association based on component and signal to noise component, receive the removing noise signal after remove the noise component.

According to each IMF component under different stress level and the original signal correlation between number calculation results, the IMF1 and IMF2 are significant correlation with the original signal, the IMF3 is real related with the original signal, and the IMF4 IMF8 are micro related with the original signal. In order to show the AE signal of each IMF component variance contribution rate and the correlation coefficient, take the IMF component as the abscissa, draw the picture of the point 1, 2, 3, 7 under the four stress level variance contribution rate and the value of correlation coefficient, as shown in Fig 4. Each IMF component variance contribution rate and relations change trend is very close to each other, there is a big correlation. The IMF 1 to IMF 4 component can represent the characteristic of the original signal, it can be used to analysis the AE signal of rock under different stress level of the characteristic, and it also reflect the EMD method is based on the intrinsic characteristics of the signal itself to adaptively decompose.

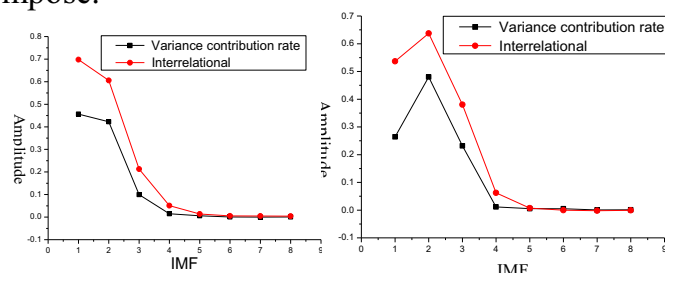



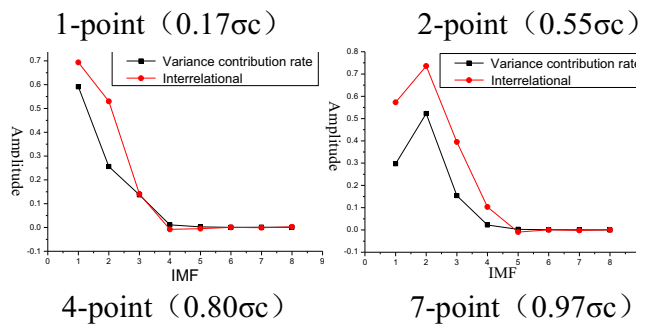

Figure 3. The IMF component variance contribution ratio and correlation coefficient of the AE signal singularity

\section{Discussion}

The marginal spectrum is integral of the timeline of the Hilbert spectrum, and is also an overall measure of each frequency component amplitude (or energy). Its accumulated amplitude of signal in probability sense, and it reflects the signal amplitude with the frequency in the whole frequency. When certain frequency energy appeared, it means there must be a vibration wave ${ }^{[14]}$.
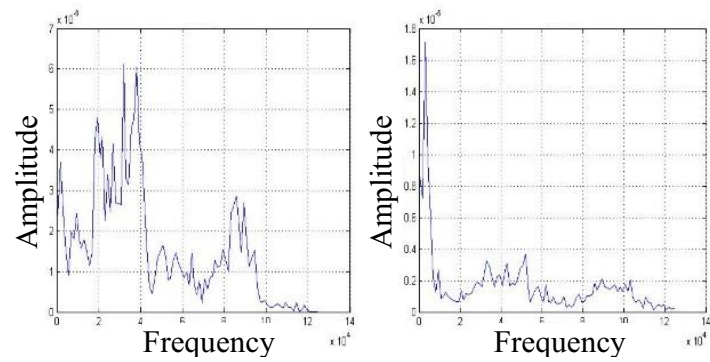

(a) 1-point

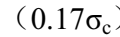

) (b)

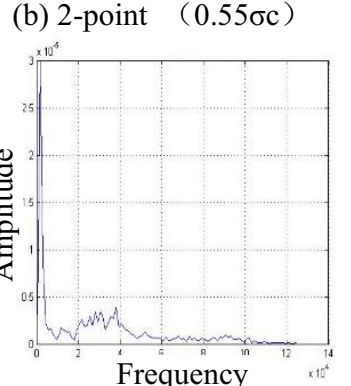

4-point $(0.80 \sigma c)$
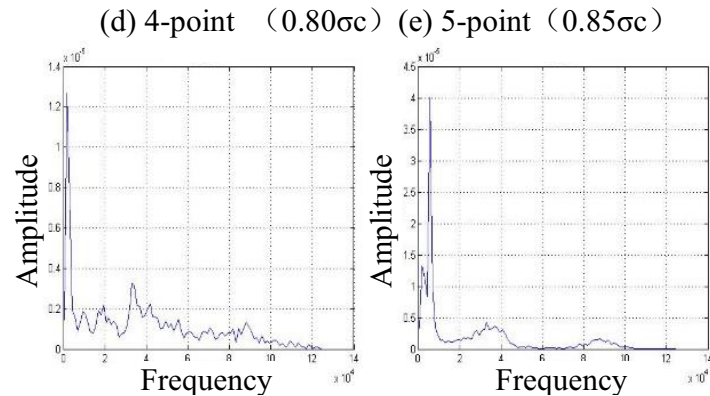

(c) 3-point $\left(0.65 \sigma_{c}\right)$

(f) 6-point $(0.95 \sigma c)$

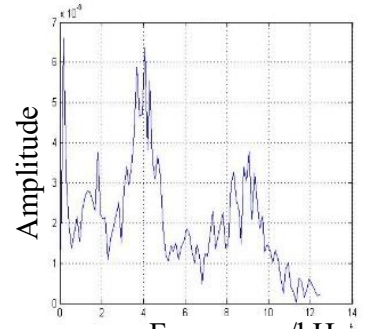

Frequency $/ \mathrm{kHz}$

(g) 7-point $(0.97 \sigma c)$

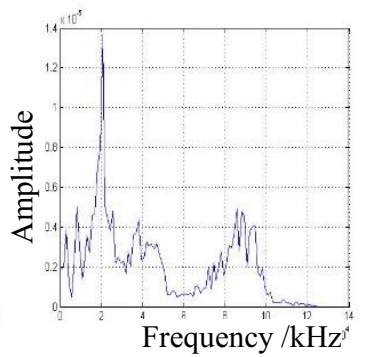

(h) C-point $(\sigma c)$

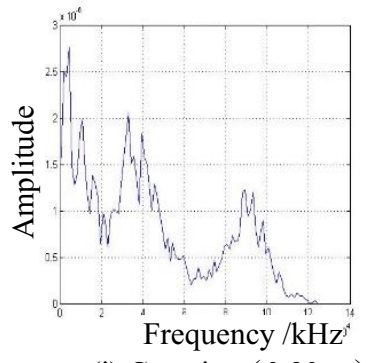

(i) $\mathrm{C}_{0}$-point $(0.90 \sigma \mathrm{c})$

Figure 4.The singular point $1 \sim \mathrm{C} 0$ marginal spectrum

Figure 4 shows the singular point $1 \sim \mathrm{C} 0$ marginal spectrum: the AE signal of the frequency range of siltstone in the process of uniaxial compression is $0 \sim 120$ $\mathrm{kHz}$. The different frequency component of AE singular signal point has different characteristics. According to the frequency peak position, the frequency range can be divided into three different frequencies: the firstly band $(0 \sim 20 \mathrm{kHz})$, corresponding to three component of IMF2 IMF 4 . The secondly band $(60 \mathrm{kHz} \sim 120 \mathrm{kHz})$, just as the IMF1 component. The thirdly frequency band $(0 \sim 100$ $\mathrm{kHz}$ ), the average peak frequencies were $3.41 \mathrm{kHz}, 38.03$ $\mathrm{kHz}$ and $85.34 \mathrm{kHz}$.

The bigger marginal spectrum amplitude makes the bigger energy. We can explore the evolution of the $\mathrm{AE}$ signal frequency in the process of rock failure by peak frequency changes in each frequency band.Analysis by above knowable, the AE signal frequency of siltstone in the rupture process of $0 \sim 120 \mathrm{kHz}$, and noise frequency band is $0 \sim 20 \mathrm{kHz}$. The AE signal of rock and noise frequency has overlap part, it means that when analyzing the characteristic of the failure process of the AE signal, we should eliminate noise interference first, and make an important theoretical basis to choose the corresponding resonance frequency of the AE sensor provides.

\section{Summary}

(1) The analysis of the EMD decomposition of HHT is a kind of adaptive decomposition method based on principal component analysis. The primary and secondary components can be determined by analysis the AE signal singularity in the process of loading.

(2) The AE signal of siltstone can be divided into 8 IMF components by the EMD decomposition. The IMF1 IMF 4 components are the main ingredient, and are the main part of the AE source. The IMF5 IMF8 for low frequency noise signal, and it should be removed in the AE signal analysis.

(3) The Frequency of AE rupture process can be divided into three main frequencies. The frequency band of $0 \sim$ $20 \mathrm{kHz}$, the second band of $20 \mathrm{kHz} \sim 60 \mathrm{kHz}$, and the third band of $60 \mathrm{kHz} \sim 120 \mathrm{kHz}$. The firstly band is overlap with noise frequency, it's greatly influenced by the noise, so the secondly and thirdly can be seen as the effective frequency. 


\section{Acknowledgements}

This work was financially supported by the Nature Science Type of Youth in education department of Heibei Province (QN2014067). All these are gratefully acknowledged.

\section{References}

1. M.C He, J.L Miao, J.L Feng. Rock burst process of limestone and its acoustic emission characteristics under true-triaxial unloading conditions [J]. International Journal of Rock Mechanics and Mining Sciences, 2010, 47(2):286-298.

2. V.A Mansurov. Acoustic emission from failing rock behaviour [J]. Rock Mech. Rock Engng., 1994, 27(3):173-182.

3. V Rudajev, J Vilhelm, T Lokajicek. Laboratory studies of acoustic emission prior to uniaxial compressive rock failure $[\mathrm{J}]$. International Journal of Rock Mechanics and Mining Sciences, 2000, 37(4):699-704.

4. P.P Nomikos, K.M Sakkas, A.I Sofianos. Acoustic emission of Dionysos marble specimens in unixaxial compression [C]. The 12th International Congress on Rock Mechanics of the International Society for Rock Mechanics. [S.1.]: [s.n], 2011:771-775.

5. P Ganne, A Vervoort, M Wevess. Quantification of pre-peak brittle damage: correlation between acoustic emission and observed micro-fracturing [J]. International Journal of Rock Mechanics and Mining Sciences, 2007, 44(5):720-729.

6. $\mathrm{T}$ Ishida, $\mathrm{T}$ Kanagawa, $\mathrm{Y}$ Kanaori. Source distribution of acoustic emissions during an in-situ shear test: implications for an analog model of seismogenic faulting in an inhomogeneous rock mass [J]. Engineering Geology, 2010, 110(3/4):66-76.

7. H Moriya, T Fujita, H Niitsuma, et al. Analysis of fracture propagation behavior using hydraulically induced acoustic emissions in the Bernburg salt mine, Germany [J]. International Journal of Rock Mechanics and Mining Sciences, 2006, 43(1):49-57.

8. Huang N E, Shen Z, Long S R, et al.The empirical mode de-composition and the Hilbert spectrum for nonlinear and non-stationary time series analysis[J]. Proceedings of the Royal Society, 1998, A: 903-995.

9. HUANG Da-ji, ZHAO Jinping, SU Jilan. Practical implementation of the Hilbert-Huang Transform algorithm $[\mathrm{J}]$. Acta Oceanologica Sinica, 2003, 25(1) :1-11.

10. Zhang Yiping, Li Xibin, Zuo Yujun. HHT analysis of blasting vibration and its application [M]. Metallurgical Industry Press, 2008.

11. XIE Heping, CHEN Zhonghui. Rock mechanics[M]. Beijing: Science Press,2004:7-14.

12. CAI M, KAISER $\mathrm{P} K$, MORIOKA $\mathrm{H}$, et al. FLAC/PFC coupled numerical simulation of AE in large-scale underground excavations [J].International Journal of Rock Mechanics and Mining Sciences,2007,44(4):550-564.
13. SHIOTANI T, OHSTU M, IKEDA K. Detection and evaluation of AEwaves due to rock deformation[J]. Construction Building Materials, 2001, 15(5/6):235-246.

14. Y.M Zhong, S.R Qin. Study on the marginal spectrum in Hilbert-Huang transform [J]. Systems Engineering and Electronics, 2004, 26:1323-1326. 